\title{
The Novel Protease-Activated Receptor 1 Antagonist Vorapaxar as a Treatment for Thrombosis in Afibrinogenemia
}

\author{
Bernard Tawfik, MD ${ }^{1}$ Elizabeth Pollard, MD ${ }^{1}$ \\ ${ }^{1}$ Division of Hematology and Oncology, University of Texas \\ Southwestern, Dallas, Texas
}

Semin Thromb Hemost 2018;44:404-406.

\author{
Yu-Min Shen, $\mathrm{MD}^{1}$
}

Address for correspondence Bernard Tawfik, MD, 745 Sid Rich Drive, Irving, TX 75039 (e-mail: bernard.tawfik@phhs.org).

Afibrinogenemia is a rare disorder characterized by the absence of detectable fibrinogen due to genetic mutations. ${ }^{1}$ The common presentation is spontaneous or unusual bleeding with minor trauma. But the recent case report and review by Santoro et al highlights that many patients present with thrombosis with or without bleeding, representing challenging clinical situations. ${ }^{2}$ Here, we add insight into management of these patients with a case of a patient with afibrinogenemia who developed recurrent arterial stenosis refractory to dual antiplatelet therapy and fibrinogen replacement treated with the novel protease-activated receptor 1 (PAR-1) antagonist vorapaxar.

Fibrinogen is a glycoprotein that is produced in the liver and stored in platelet $\alpha$ granules after uptake from plasma. ${ }^{1}$ Fibrinogen functions in fibrin clot formation, thrombin regulation, platelet aggregation, and fibrinolysis. Afibrinogenemia is an autosomal recessive disorder associated with a quantitative deficiency of fibrinogen and a prevalence of 1 in $1,000,000 .^{3}$ The clinical manifestations of afibrinogenemia include umbilical cord hemorrhage and bleeding in the gastrointestinal (GI) tract, genitourinary tract, mucosa, skin, and the central nervous system. ${ }^{4}$ Approximately $30 \%$ of patients with afibrinogenemia may also have thrombotic complications due to the antithrombin function of fibrinogen. Rarely patients may have both bleeding and thrombotic complications related to the disease. ${ }^{5,6}$ Treatment for hemorrhage includes antifibrinolytics and/or fibrinogen replacement therapy with fresh frozen plasma, cryoprecipitate, and plasma-derived fibrinogen concentrate. ${ }^{7}$ In the setting of a thrombotic phenotype, there is no clear consensus, but treatment with low molecular weight heparin, antiplatelet agents, vasodilators, lepirudin, and direct thrombin inhibitors with fibrinogen replacement therapy has been used. ${ }^{8}$ We present a case of a patient with bleeding and thrombotic complications associated with congenital afibrinogenemia treated with the novel PAR-1 antagonist vorapaxar (Zontiv- ity, Aralez Pharmaceuticals Inc.) and fibrinogen replacement therapy (-Fig. 1).

The patient is a 34-year-old Hispanic woman with a past medical history of sensorineural hearing loss, type 1 renal tubular acidosis, GI bleed, and congenital afibrinogenemia. The patient's history of afibrinogenemia was associated with episodes of gingival bleeding, hemarthrosis, menorrhagia, and hematemesis. She was treated with on-demand infusions of cryoprecipitate for many years with control of symptoms. In April 2010, she began having recurrent painful edema in her right great toe that progressed to her other digits and foot and was relieved with cryoprecipitate infusions. Over the next year, the pain became more persistent. Evaluation for ischemia with an angiogram demonstrated stenosis of the infrarenal aorta and narrowing of the dorsalis pedis artery. In June 2011, she was started on fibrinogen concentrate infusion to reduce the risk of bleeding intraoperatively and underwent endovascular aortic repair with endoprosthetic stents placed to dilate the distal aortic stenosis. She was treated with clopidogrel $75 \mathrm{mg}$ orally (PO) once daily (QD), aspirin $81 \mathrm{mg}$ PO QD, and prophylactic fibrinogen concentrate infusions $2000 \mathrm{mg}$ IV (intravenous) once weekly due to the history of GI bleeding. She developed recurrent microembolic phenomena after cessation of clopidogrel, which was restarted. In July 2016, she presented with complaints of cramping lower extremity pain on exertion that had progressed to pain at rest and was admitted for critical limb ischemia. Angiogram of the infrarenal abdominal aorta revealed multifocal hypodense plaques in the proximal third of the stents bilaterally consistent with mild-to-moderate in-stent stenosis (-Figs. 2 and 3). She then underwent bilateral common femoral artery exploration with kissing balloon angioplasty with resolution of the stenosis and patent bilateral lower extremities postprocedure. After discharge from the hospital, there was grave concern that she had developed in-stent stenosis while on published online May 15, 2018
Issue Theme Recent Advances in Thrombosis and Hemostasis - Part III; Guest Editor: Sam Schulman, MD, PhD.
Copyright $\odot 2018$ by Thieme Medical Publishers, Inc., 333 Seventh Avenue, New York, NY 10001, USA. Tel: +1(212) 584-4662.
DOI https://doi.org/ $10.1055 / \mathrm{s}-0038-1654715$. ISSN 0094-6176. 


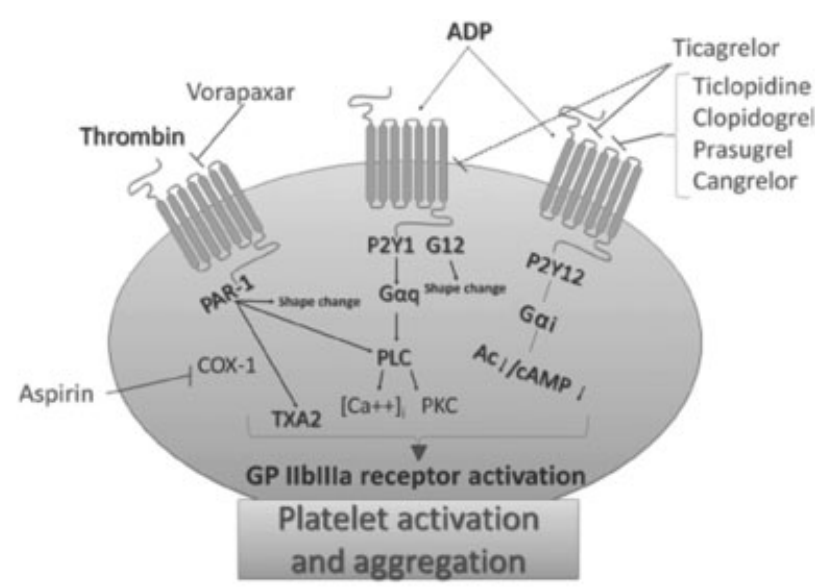

Fig. 1 Multiple agents inhibit platelet activation but only vorapaxar inhibits protease-activated receptor 1 (PAR-1). Platelet activation pathways and drug molecular targets. Thrombin binds to PAR-1, which leads to shape change, phospholipase $C$ (PLC) activation, thromboxane $A 2(T \times A 2)$ generation, and activation of the glycoprotein (GP) IIb/IIla receptor, resulting in sustained platelet aggregation. Cyclooxygenase 1 (COX-I) catalyzes the production of $T \times A 2$, a potent platelet aggregator, generated by platelets activated by thrombin and other agonists. Adenosine 5'-diphosphate (ADP) binds to its 7-transmembrane domain receptors, P2Y1 and P2Y12, to activate platelets. P2Y1 is coupled to Gaq and G12. Gaq is linked to a signaling pathway involving PLC activation, resulting in a rise in the intracellular calcium concentration ([Ca +2$] \mathrm{i})$ and protein kinase $\mathrm{C}$ (PKC) activation, leading to GP IIb/IIla activation and transient platelet aggregation. G12 mediates platelet shape change. P2Y12 is linked to Gai-coupled signaling cascades associated with adenyl cyclase (Ac) downregulation and decreased cyclic-3',5'-monophosphate (cAMP) production, which mediates GP IIb/IIla receptor activation, leading to sustained platelet aggregation. (Reproduced with permission of $\mathrm{Di}$ Minno MND, Guida A, Camera M, Colli S, Di Minno G, Tremoli E. Overcoming limitations of current antiplatelet drugs: a concerted effort for more profitable strategies of intervention. Ann Med 2011;43 (7):531-544, under open access license https://creativecommons. org/licenses/by/2.0/legalcode with no alterations.)

clopidogrel and aspirin. Clopidogrel was discontinued and she was started on vorapaxar $2.08 \mathrm{mg} P O \mathrm{QD}$, and the same dose of aspirin was continued. Surveillance angiogram 6 and 12 months after starting vorapaxar revealed patent infrarenal stents bilaterally and improved multifocal plaques

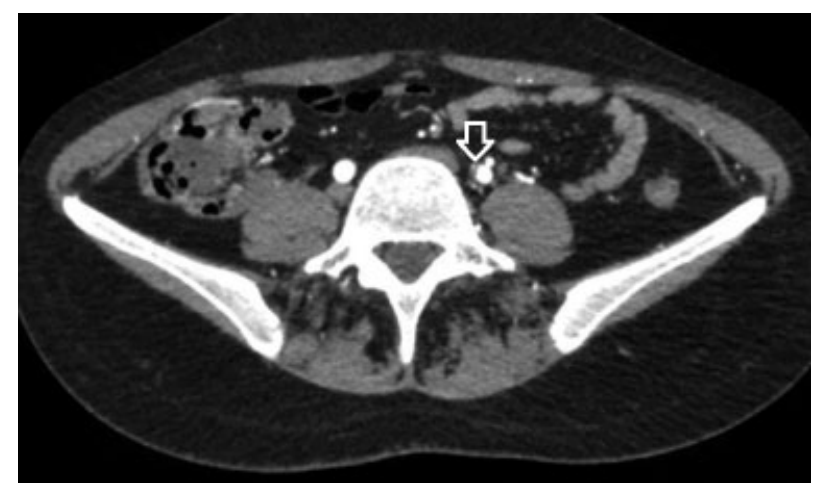

Fig. 2 Intra-abdominal stents with left common iliac stenosis (White arrow). in the left and right common iliac arteries. The plan was to discontinue vorapaxar after 12 months of therapy, but given patient's response and tolerability to the therapy, it has been continued to date.

Treatment recommendation per the 2016 Delphi consensus in patients with bleeding phenotype of afibrinogenemia is to start secondary prophylaxis after first life-threatening bleeding episode. ${ }^{8}$ Fibrinogen replacement therapy is a risk factor for thromboembolism, although the exact incidence of events associated with the treatment is unknown. In patients with thrombosis, fibrinogen replacement therapy should be administered concurrently with low molecular weight heparin. For patients on antiplatelet therapy, fibrinogen replacement should be considered based on the risk of bleeding. Our patient had a history of GI bleeding and therefore she was continued on replacement therapy for the duration of antiplatelet therapy. The proposed mechanism for the development of thrombosis in patients with congenital afibrinogenemia is lack of antithrombin I activity of fibrin. Normally, this activity of fibrin includes sequestering free thrombin, which downregulates thrombin activity and decreasing subsequent platelet activation. ${ }^{9}$ But in patients with afibrinogenemia, free thrombin is not sequestered, and increasing levels of free thrombin lead to platelet activation through the PAR- 1 and a prothrombotic state. ${ }^{10}$ In addition, increasing thrombin promotes proliferation of vascular smooth muscle cells, which may have contributed to stenosis in this patient. PAR-1 is found on the surface of platelets and vascular endothelium. Thrombin levels were not measured in this patient, but her episodes of limb pain improved with fibrinogen replacement therapy, which highlights the possibility of elevated thrombin activity as the cause and reduction of its activity when fibrinogen was replaced.

Vorapaxar is a competitive and selective antagonist of PAR-1 thrombin receptor on platelets. In a randomized, double-blind, placebo-controlled trial (TRA2 ${ }^{\circ}$ P-TIMI 50) of vorapaxar, patients with a history of myocardial infarction, ischemic stroke, or peripheral arterial disease (PAD) were randomized to vorapaxar or placebo. ${ }^{11}$ Vorapaxar reduces acute limb ischemia in patients with symptomatic PAD. Our patient was started on vorapaxar as an antiplatelet therapy for increased thrombin activity, which was thought to be causing thrombosis and stenosis. If the development of thrombosis in patients with congenital afibrinogenemia is secondary to excess thrombin, then blockade of PAR-1 with this new antiplatelet agent would be a rational therapy. As there is an increased risk of bleeding with the antiplatelet agents, fibrinogen replacement would need to be determined on a case-by-case basis. While vorapaxar activity on platelet aggregation has been evaluated, the effect on endothelial cells and smooth muscle cells has not been studied and is an area for further research.

In conclusion, vorapaxar targets the thrombin pathway and is a reasonable treatment option for refractory thrombosis in patients with afibrinogenemia. Further research will need to focus on management strategies of these complex patients. 


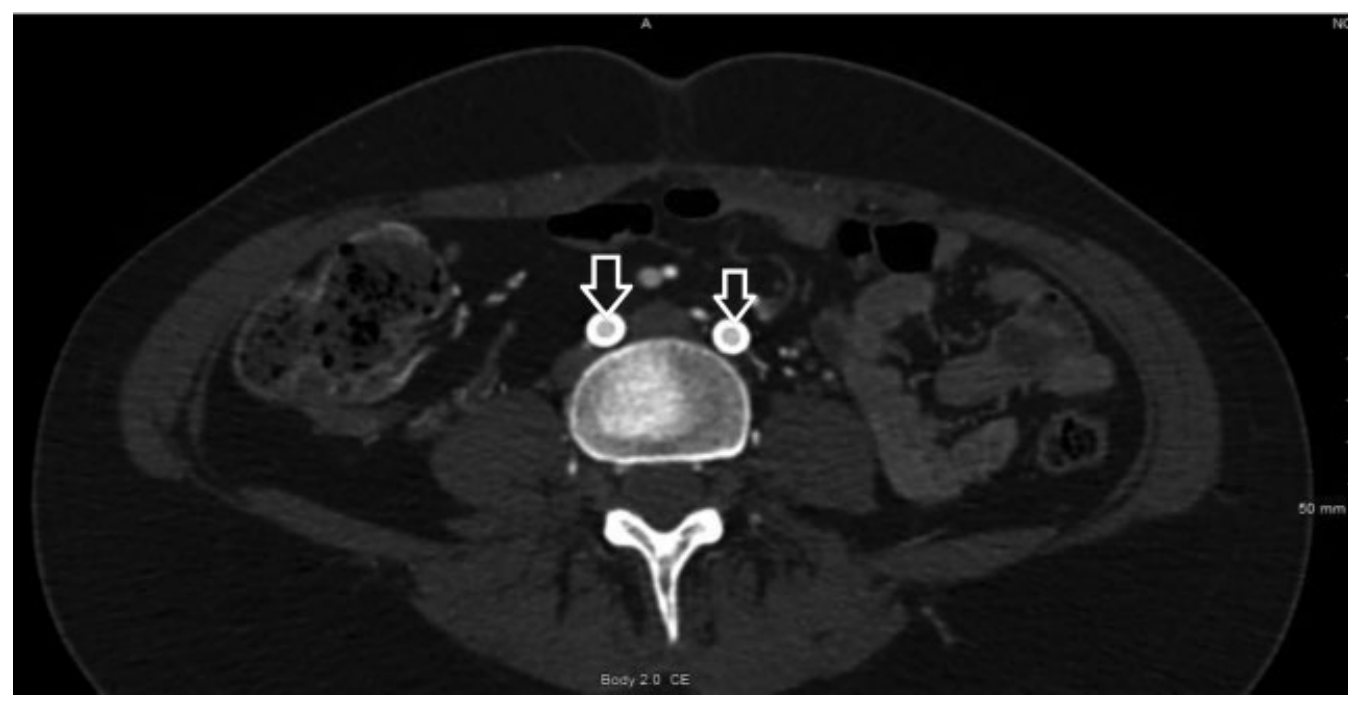

Fig. 3 Bilateral intra-abdominal stent stenosis (White arrows).

\section{Conflict of Interest}

The authors have no conflict of interest to disclose.

\section{References}

1 Acharya SS, Dimichele DM. Rare inherited disorders of fibrinogen. Haemophilia 2008;14(06):1151-1158

2 Santoro C, Massaro F, Venosi S, et al. severe thrombotic complications in congenital afibrinogenemia: a pathophysiological and management dilemma. Semin Thromb Hemost 2016;42(05): 577-582

3 Asselta R, Duga S, Tenchini ML. The molecular basis of quantitative fibrinogen disorders. J Thromb Haemost 2006;4(10): 2115-2129

4 Peyvandi F. Epidemiology and treatment of congenital fibrinogen deficiency. Thromb Res 2012;130(Suppl 2):S7-S11

5 Chevalier Y, Dargaud Y, Argaud L, Ninet J, Jouanneau E, Négrier C. Successive bleeding and thrombotic complications in a patient with afibrinogenemia: a case report. Thromb Res 2011;128(03): 296-298
6 Mukaddam A, Patil R, Jadli A, Chandrakala S, Ghosh K, Shetty S. Paradoxical bleeding and thrombosis in a patient with afibrinogenemia and fibrinogen Mumbai mutation. Am J Clin Pathol 2015; 143(05):755-757

7 Bornikova L, Peyvandi F, Allen G, Bernstein J, Manco-Johnson MJ. Fibrinogen replacement therapy for congenital fibrinogen deficiency. J Thromb Haemost 2011;9(09):1687-1704

8 Casini A, de Moerloose P; Congenital Fibrinogen Disorders Group. Management of congenital quantitative fibrinogen disorders: a Delphi consensus. Haemophilia 2016;22(06):898-905

9 de Bosch NB, Mosesson MW, Ruiz-Sáez A, Echenagucia M, Rodriguez-Lemoin $A$. Inhibition of thrombin generation in plasma by fibrin formation (antithrombin I). Thromb Haemost 2002;88(02): 253-258

10 Korte W, Poon M-C, Iorio A, Makris M. Thrombosis in inherited fibrinogen disorders. Transfus Med Hemother 2017;44(02): 70-76

11 Bonaca MP, Scirica BM, Craeger MA. Vorapaxar in patients with peripheral artery disease: results from TRA2 ${ }^{\circ} \mathrm{P}-\mathrm{TIMI}$ 50. Circulation 2013;127(14):1522-1529 